\title{
El laboratorio de la novela: instrumentos y pruebas de una voz autoral en The Golden Notebook, de Doris Lessing
}

INÉS GARCÍA

Durham University

\begin{abstract}
Resumen
Este artículo propone una lectura feminista de The Golden Notebook, de Doris Lessing, a partir de teorías narrativas enfocadas a la estructura metaficcional de un texto y la función de la narradora como autora de la misma novela. El análisis de los paratextos, que dividen los distintos niveles narrativos dentro de la novela misma, pretende revelar el ejercicio autoral de la narradora, que consiste en una búsqueda del lenguaje adecuado para escribir su historia. Este estudio narratológico, además, permite profundizar en la complejidad estructural de esta novela, que entreteje niveles narrativos y ejercicios de distintas voces autorales directamente enfocados al acto de escritura.
\end{abstract}

Palabras clave: metaficción, novela inglesa, Lessing, paratextos, feminismo

\begin{abstract}
This paper offers a feminist reading of The Golden Notebook, by Doris Lessing, through narrative theories focused on the metafictional structure of texts and the narrator's role as author of that very novel. The analysis of paratexts - which evince different narrative levels within the novel-aims to reveal the narrator's authorial exercise, characterized by the search of an adequate language to write her own story. This narratological approach goes deep into the structural complexity of this novel, interwoven by several narrative frames and the practice of different authorial voices directly committed to the act of writing.
\end{abstract}

Keywords: metafiction, English novel, Lessing, paratexts, feminism 


\section{García, Inés. "El laboratorio de la novela" Poligrafías, Nueva época, no. 4 | noviembre 2018}

Never to be yourself and yet always - that is the problem.

-Virginia Woolf, "The Modern Essay"

Cómo leer aquellas novelas en las que el narrador se refiere de manera obsesiva y deliberada a la historia que nos cuenta dándose una autoridad narrativa que no le correspondería si fuera "sólo" el narrador? ¿Cómo reaccionar ante un narrador que no sólo se adjudica una autoridad narrativa sino una autoridad creativa? ¿Cómo impacta en nuestra lectura que el narrador, en primera persona, nos diga que es un escritor o incluso un novelista? Aunque toda obra literaria conjuga, a su manera, tema y forma $-\mathrm{y}$ en este sentido se puede hablar de un asunto de grado presente en mayor o menor medida-, en una novela consciente de su forma tenemos la insistencia, por parte del narrador, de que uno de los temas principales de su historia es el proceso mismo de escritura. De acuerdo con Linda Hutcheon, cuando existe "a dual interest in the storytelling as well as in the story told" (1980: 37), estamos frente a una novela autorreflexiva o narcicista.

Algunos rasgos narrativos que comparten las novelas autorreflexivas y que analizaré en The Golden Notebook (1962), de Doris Lessing, son, por ejemplo, el intercambio de voces narrativas y su justificación; una teorización del género novelístico y, en ocasiones, autobiográfico; la presencia de un metatexto a través del cual el narrador comenta la historia; una conciencia autocrítica del uso del lenguaje en tanto vocabulario, estilo y tono; y, finalmente, la construcción identitaria del narrador como novelista a través de la exhibición de la maquinaria narrativa o lo que Steven Kellman llamó el "laboratorio de la novela" (1980: 4): el "behind the scenes of th[e] book" (Fowles, 2010: 98). Así, la novela autorreflexiva es un tipo de literatura del yo, pero, más que un afán autobiográfico, tiene como objeto de estudio al artista en su calidad de artista, es decir, en su momento de creación. La novela autorreflexiva es, entonces, la literatura del yo que escribe.

The Golden Notebook es la historia del proceso de creación de una novela: la misma que tenemos en las manos. Tanto en Free Women, una novela narrada en 
tercera persona, como en los Cuadernos, e indirectamente en todo el metatexto, el tema que hila la narración es la vida de Anna Wulf, protagonista, narradora y autora de la novela, vista desde su relación con la sociedad en un contexto muy específico - la década de 1960 y sus alrededores - y, por supuesto, con ella misma. La división temática de los Cuadernos constituye el primer intento de la escritora de uniformar y reconciliar sus memorias: el Cuaderno negro retrata sus experiencias de juventud en África; el Cuaderno rojo relata su pertenencia al Partido Comunista y su vida política; el Cuaderno amarillo, por otro lado, consiste en un ejercicio metaficcional en el que Anna Wulf escribe una novela donde la protagonista, Ella, escribe otra novela; finalmente, el Cuaderno azul toma la forma de un diario personal. El carácter autobiográfico de The Golden Notebook, cabe aclarar, consiste sólo en la relación entre la voz narradora (Anna Wulf) y la historia que se cuenta (su vida).

La lectura de The Golden Notebook como una novela autorreflexiva arroja luz a ciertos elementos formales que de otra manera no resultan del todo justificados y que replantean la necesidad de Anna Wulf de escribir su historia. Primero, Anna no es sólo la protagonista y narradora de su historia; es una escritora. Su desarrollo como personaje, entonces, es inseparable de su definición como novelista. Segundo, existen elementos paratextuales -que rodean e interrumpen los distintos textos que se nos presentan- que acentúan la importancia y función del aparato novelístico. Tercero, se exploran las posibilidades del artificio: el cambio indiscriminado de voces narrativas, por ejemplo, sugiere un interés que va más allá del ánimo realista - contar la experiencia como pasó- y sugiere un ejercicio de búsqueda autoral.

The Golden Notebook es un ejercicio, un ensayo de las experiencias de Anna, la narradora, en el que ella trata de dar significado al mundo y a ella misma. Pero no es sólo que no sepa cómo plantear ese significado porque el lenguaje sea inadecuado, sino que ella misma no sabe cuál es: la memoria, jueza implacable, se manifiesta siempre. El reto consiste en representar la experiencia a partir de una construcción narrativa, es decir, artificial y artística. La búsqueda identitaria de Anna está limitada a su voz autoral; es decir, a quién es Anna la escritora, qué tipo de escritora pretende ser y por qué tiene problemas para escribir como quisiera. 
The Golden Notebook es el laboratorio de la novela donde, más que el resultado definitivo, importa el experimento a prueba y error de las voces narrativas de Anna a través de las cuales intenta no sólo definirse como la escritora que quiere ser, sino plasmar, de la manera más franca posible, el significado de su experiencia. El material principal de este experimento son los paratextos, ${ }^{1}$ a través de los cuales se expresan las distintas voces de Anna y se configura un espacio de intercambio y posibilidades narrativas.

Debido a la complejidad formal de esta novela, se pueden considerar elementos paratextuales a partir de la presencia de por lo menos tres textos: Free Women, "The Notebooks" y el metatexto (notas autorales), que analiza y critica lo que cuentan los cuadernos. Al tener tres tipos distintos de textos claramente diferenciados, la intratextualidad se vuelve no sólo una herramienta de lectura sino un tema, pues la relación de la voz autoral con su(s) texto(s) es la característica principal de una novela autorreflexiva.

En esta novela los paratextos que dividen e integran las realidades ficcionales toman la forma de peritextos indicativos -intertítulos e intervenciones entre corchetes - y fungen como un espacio experimental en el que la voz autoral se ejercita como autoridad narrativa de su novela. Así, el uso de corchetes antes de entrar a uno de los cuadernos le permite a Anna ubicarse en el lugar de una tercera persona y, como consecuencia, tener más de una perspectiva; el comentario explicativo sobre una experiencia que acaba de narrar la obliga a tomar una postura, muchas veces moral, que de otra manera pasaría desapercibida o nunca sería asumida. Finalmente, el uso de distintos géneros literarios para conformar su novela la lleva a un ejercicio artístico no sólo en cuanto la conciencia de la autonomía de la ficcionalidad sino en las posibilidades del artificio. Los

1 De acuerdo con Genette, los paratextos son aquellos elementos inseparables del texto que, no obstante, se encuentran fuera de él. "Aquello por lo cual un texto se hace libro y se propone como tal a sus lectores. Se trata aquí de un umbral o - según Borges- de un 'vestíbulo' que ofrece la posibildidad de entrar o retroceder" (2001: 7). Un peritexto, entonces, consiste en la separación de distintos niveles textuales dentro de un mismo volumen (2001: 290). A partir de ahora, me referiré indistintamente a paratexto o peritexto, pues todos los elementos aquí mencionados forman parte del libro como objeto y, más importante aún, de la historia. 
paratextos, entonces, son el instrumento principal en el laboratorio novelístico de Anna, aquél en el que se gesta su voz autoral.

La primera función de esta división formal es por supuesto la de tomar postura ante la ficción y reconocer el artificio. Esto significa no ser indiferente al hecho de que se nos presenta, sin velos, la calidad ficticia de aquello que habíamos firmado como un contrato de lectura convencional y que cambia a un pacto metaficcional entre narradora y lectora. La intención estética de introducir un marco metaficcional es recordar que lo que se está leyendo es una obra ficcional en sí misma tanto como la que se cuenta dentro de ella y hacer un comentario acerca del proceso creativo.

En The Golden Notebook, como en muchas novelas autorreflexivas, la metaficción juega un papel determinante y tiene como principal herramienta los paratextos, que no sólo revelan la existencia de las diferentes realidades ficcionales, sino que median entre ellas armando, en cada intervención, una voz autoral superior a la supuesta tercera persona que narra "Free Women: 1" y a la voz en primera persona que se le otorga a Anna en los Cuadernos. La posibilidad de que Anna sea la voz autoral de ambos textos, Free Women y "The Notebooks", se sugiere desde el principio y se comprueba cuando Anna decide escribir una novela que se llama The Golden Notebook y cuya primera oración es la que nosotros ya leímos.

El cambio de voces, resultado del salto entre planos ficcionales, se multiplica al igual que los planos y sus voces locutoras. Consideremos, por ejemplo, el intertítulo que aparece en el Cuaderno amarillo: The Shadow of the Third, que da nombre a la novela de Ella, personaje principal de una de las novelas de Anna Wulf. La relación entre el cambio de contratos de lectura y voces locutoras resulta mucho más compleja que aquella definida como el mise en abyme. La insistencia en el cambio de voces y en personajes que se encarnan en autoras revela una conciencia y preocupación por el proceso creativo que se puede atribuir a la voz autoral de la novela como unidad y que, por tanto, modula al resto. Los elementos paratextuales implican y exhiben una voz superior que coordina lo que se va a escribir a continuación. La variedad de estas formas paratextuales es, entonces, un ejercicio constante del proceso creativo de una 
autoridad literaria. En The Golden Notebook, como toda self-begetting novel, ${ }^{2}$ esta voz autoral es Anna.

Una de las principales formas paratextuales con las que nos encontramos en The Golden Notebook son los intertítulos. La primera diferencia entre el título de la obra y sus intertítulos es que los segundos están dirigidos a un público más reducido (aquellos que ya empezaron a leer la novela) y establecen una familiaridad entre lo que los precede y lo que los sucede (Genette, 2001: 294). Cuando nos encontramos con el final de la primera parte, "Free Women: 1", y la presencia de un nuevo intertítulo en la misma hoja, llamado "The Notebooks", se establece la división entre dos realidades ficcionales: la de Free Women como el texto madre y la que crea Anna Wulf, “The Notebooks". A pesar de que nos encontramos con los Cuadernos en la primera parte de la novela, no es sino hasta el intertítulo que el marco metaficcional se revela, pues la sola mención a lo que escribe Anna no basta para crear ese segundo espacio; se necesita tener acceso a los Cuadernos, leerlos directamente.

Al ser un espacio de transacción, es decir, de intercambio entre la voz autoral y la lectora, los paratextos actúan inevitablemente sobre nuestra interpretación. Así, el subtítulo “The Notebooks" señala la continuación de una parte distinta a la anterior y, más importante, establece un nuevo contrato de lectura. La lectora no sólo se vuelve consciente de que está a punto de empezar a leer los cuadernos de Anna Wulf, sino que acepta los términos de un contrato metaficcional: sabe que lo que va a leer ha sido creado por un personaje de la novela.

La función de los intertítulos en The Golden Notebook está, pues, directamente relacionada con los cambios de contrato de lectura, con los que la lectora se enfrenta a lo largo de la novela. Como sabemos, las cinco partes en las que se divide la novela Free Women están a su vez divididas entre la narración

2 La diferencia entre una novela autorreflexiva y una self-begetting novel es, una vez más, un asunto de grado, y muchas veces se podrá poner a discusión si el momento referido por Kellman (el instante en que el novelista toma la pluma y empieza a escribir su novela) está sólo sugerido o se relata abiertamente. Sí hay que destacar, no obstante, que si bien no todas las novelas autorreflexivas son self-begetting, todas las self-begetting son exhaustivamente autorreflexivas. 
heterodiegética, que corresponde a "Free Women 1-5", y los Cuadernos, que siempre son la segunda parte, es decir, cierran cada capítulo, narrados en voz de Anna pero introducidos por una aparente tercera persona que habla entre corchetes, signo de puntuación que por excelencia denota una voz individual: "[The four notebooks were identical, about eighteen inches square, with shiny covers, like the texture of a cheap watered silk]" (Lessing, 1999b: 54). El segundo efecto de los intertítulos, o paratextos indicativos, entonces, es el cambio de voz narrativa. Si en el texto madre tenemos una voz heterodiegética que narra parte de la historia de Wulf, sabemos (porque se nos ha anticipado) que la voz locutora de los Cuadernos es la misma Anna: "Every time I sit down to write" (54).

No obstante, no basta con decir que el cambio de voces es el salto de tercera a primera persona. Si fuera la misma tercera persona que narró "Free Women: 1 " y que ahora va a presentarnos los Cuadernos, ¿por qué la necesidad de intervenir entre corchetes? Probablemente sea lógico decir que una vez introducido el intertítulo "The Notebooks", que abre el espacio de la voz de Anna en primera persona, una intervención de una voz distinta debe ir entre corchetes para diferenciarse del yo que enunciará los Cuadernos, pues está "fuera" de éstos. Sin embargo, esta multiplicación de voces no resulta del todo justificada ni pertinente si se piensa que ya en la parte anterior se nos había contado de los diarios; más aún, si aceptamos desde el principio la hipótesis de que Anna es la única voz locutora del texto, ¿por qué el desdoblamiento de dos terceras personas aparentemente distintas?, - distintas porque desempeñan distintos papeles-. Mientras que la primera tercera persona, que se encarga de narrar las cinco partes de Free Women, se concentra en contar la historia de vida de Anna y en desarrollar el espacio, el tiempo y los personajes de este relato, la voz entre corchetes se enfoca en una descripción breve pero puntual de lo que vamos a leer a continuación y de cómo está escrito. Es decir, consiste en el retrato más involuntario de la lucha de Anna con el lenguaje, pues hace referencia a garabatos y rayas negras al final de una parte, todavía sin criticarlas, y, más importante, al estilo de la letra de Anna, que cambia constantemente.

Se puede decir que esta voz, distanciada de Anna la novelista y de Anna la autobiógrafa, se personifica como la mayor autoridad literaria, pues ya leyó los 
Cuadernos y considera pertinente explicarlos: "[And this is what had happened. The first book, the black notebook, began with doodlings, scattered musical symbols, treble signs that shifted into the $£$ sign and back again; then a complicated design of interlocking circles, then the words:]" (54). La autoridad de esta voz, si bien no empieza desveladamente, se refleja en que ella decide qué nos anticipa y qué cuentan los Cuadernos por sí mismos.

Sabemos, entonces, que aunque tenemos un espacio considerable dedicado a los Cuadernos (en total será más de la mitad de la novela), sí existe una voz autoral que decide deliberadamente qué se muestra y qué no. Así, el retrato de los Cuadernos, que parecía una copia fiel de éstos, se configura poco a poco como un proceso creativo de escritura, en el que una voz ensaya su autoridad y que además se encarga de describir el proceso pasado de escritura de los diarios: "[The whole of the above was scored through-cancelled out and scribbled underneath: No, it didn't come off. A failure as usual. Underneath was written, in different hand-writing, more neat and orderly than the long entry, which was flowing and untidy:]" (351). La voz entre corchetes, desdoblada de la misma que escribió los diarios, no sólo nos cuenta parte del proceso de escribir The Golden Notebook, sino que retrata la lucha con la hipotética primera escritura de los diarios. Desde este punto de vista, la lectora tiene acceso a dos distintos procesos creativos que se recrean con su lectura: cómo se va escribiendo The Golden Notebook y cómo escribió Anna los diarios: "[The black notebook now abandoned its original intention to be divided into two parts]" (501).

La insistencia en la variedad de estilos de la letra de Anna - y su obsesión por dividir los diarios en temas y colores- refleja la preocupación de la escritora por probar diferentes formas de escritura, que finalmente tendrán su ensayo principal en la novela, que le concede el espacio para ser distintas voces: "[Here were gummed in several sheets of ordinary lined writing paper torn off a blue writing pad, written over in very neat tidy hand-writing] (288); "[For something like eighteen months the blue notebook consisted of short entries different in style not only from previous entries in the blue notebook but from anything else in the notebooks]" (447); "[and now Anna continued in a different writing, not the clear small script of the daily entries, but fluent, rapid, in parts almost unintelligible with the speed it had been written]" (448, cursivas mías). 
Además, el cambio de estilo, que probablemente se puede relacionar con el contenido del relato, tiene un eco con una de las características principales de la novela autorreflexiva: a través de su escritura, la novelista reconoce un grado de autonomía de la ficción y aunque tenga claro lo que va a decir, nunca sabe cómo van a tomar forma las palabras y probablemente escriba cosas que no sabía que tenía que decir: "I hadn't known I'd thought it until I said it" (520). En el caso de Anna, el cambio de estilo en la letra no sólo hace evidente una afectación emocional respecto al tema o al pasaje específico de la experiencia que está contando, sino que, muchas veces, se trata de una tensión con el lenguaje que tiene para contarlo y con el poder de la palabra escrita, el cual no depende de ella: "When I write the word down and look at it, I realise its power to disturb. When I remember the three, how they were, their characters, there is no shock or moment of disturbance. But at the word homosexual, written-well, I have to combat dislike and disquiet" (72).

Si bien los peritextos indicativos configuran una conciencia y por tanto una postura autocrítica respecto a lo que Anna escribió, el mayor comentario crítico de los cuadernos tiene lugar en su mismo proceso de escritura; es decir, está camuflado en la misma narración. La relación problemática idea-instrumento/ lenguaje se devela por completo en las intervenciones deliberadas de Anna, ya en los cuadernos y por tanto en primera persona.

Una de las primeras luchas de la escritora con el lenguaje es la inadecuación del vocabulario: "I keep writing the word group. Which is a collection of people. Which one associates with a collective relationship-and it is true we met day after day for months, for hours every day. But looking back [...] to really remember what happened, it is not at all like that" (78). Otra de las batallas lingüísticas de Anna tiene lugar en la autocrítica del estilo y del tono que ha decidido usar para cierto pasaje. Así, en desacuerdo con ella misma, se queja y discute la manera en la que está retratando una escena: "I see I am falling into the self-punishing, cynical tone again. Yet how comforting this tone is", le cuenta Anna a su lectora, para luego concluir: "I'll go on with this when I can write straight, not in that tone" (86). De manera similar, aunque desde un distanciamiento temporal, Anna comenta algo que escribió varios años atrás: 
I read this over today, for the first time since I wrote it. It's full of nostalgia, every word loaded with it, although at the time I wrote it I thought I was being "objective." Nostalgia for what? I don't know. Because I'd rather die than have to live through any of that again. And the "Anna" of that time is like an enemy, or like an old friend one has known too well and doesn't want to see (145).

La palabra objetiva nos remite a un intento del relato fáctico, que no incluye perspectivas emocionales ni segundas versiones: "Matching what I had written with what I remembered it all seemed false" (455). A través de su tono, estilo y perspectiva, esto es, a través de su subjetividad, Anna se enfrenta a la evidente imposibilidad de este tipo de narración, aun si intenta, desesperadamente, probar distintas voces que le concedan una mayor autenticidad.

Junto con la inadecuación del vocabulario viene una intención-creativa- de retratar lo verdadero. El problema, no obstante, no es sólo la individualidad de la locutora ni la falta de armonía entre la palabra y la idea, sino considerar la preexistencia de lo "cierto" y lo "real" antes de narrarlo. El bloqueo creativo de Anna, si en un principio se limita a la inconformidad y al descontento en el momento de escribir, se agudiza cuando fracasa en sus innumerables intentos - más de quinientas páginas- de plasmar la experiencia como la vivió: "I realised [...] that what I 'remembered' was probably untrue" (502), nos dice Anna al reconocer la futilidad de su intento. Finalmente, después de escribir casi seiscientas páginas, Anna acepta que tiene un bloqueo: "I felt the inertia of my hand, which was cold and unable to reach out for the pen [...] the real reason is that I have a writer's block. That's all. And it's the first time I've admitted it" (574-577). La paradoja de esta declaración tiene al menos dos niveles. Por un lado, a través del lenguaje escrito, Anna nos cuenta que en ese momento no puede escribir: el carácter "real" de la razón que no le permite escribir se pone en duda cuando nos dice eso escribiéndolo. Por otro lado, concede al lenguaje escrito y a la ficción -la novela que estamos leyendo- la autoridad de discutir sobre una hipotética naturaleza de lo cierto o verdadero y lo falso: "Why a story at all-not that it was a bad story, or untrue, or that it debased anything. Why not, simply, the truth?" (61). La discusión, casi filosófica, sobre la cualidad del 
lenguaje y el distanciamiento inevitable entre sus ideas y su escritura, resulta una batalla perdida en el clímax del bloqueo de la escritora hasta ser "won by the language" (508).

La conciencia y aceptación de que el lenguaje es inadecuado para plasmar la experiencia vivida abre un espacio de diálogo entre Anna la novelista y la novela como género literario. En primer lugar, está el reconocimiento de cierta autonomía de la ficcionalidad en el acto de narrar y de la cual la autora no tiene todo el control: "It frightens me that when I'm writing I seem to have some awful second sight, or something like it, an intuition of some kind; a kind of intelligence is at work that is much too painful to use in ordinary life" (546). La tensión en la escritura de Anna se relaciona con un miedo al resultado, similar a la sensación de disgusto por no identificarse con la Anna escritora de años pasados y que la obliga a replantearse la experiencia. El sentido moral de la escritura, esto es, no ser indiferente a la ideas sugeridas y planteadas en su relato, es una obsesión primaria de Anna: "But looking back I see Mrs Boothby as a lonely pathetic figure. But I didn't think so then. I saw her as a stupid 'aborigine'. Oh, Lord, it's painful thinking of the people one has been cruel to" (135). La culpa que siente, es evidente, se debe a que se avergüenza de la persona que fue.

Por otro lado y como parte esencial del metatexto, en voz de Anna tenemos una explícita teorización sobre el género novelístico que después tomará forma en un ethos autoral, pues lo que Anna decide escribir no es un diario ni una autobiografía, sino una novela:

The point is, that the function of the novel seems to be changing; it has become an outpost of journalism; we read novels for information about areas of life we don't know [...] we read to find out what is going on. One novel in five hundred or a thousand has the quality a novel should have to make it a novel - the quality of philosophy [...] The novel has become a function of the fragmented society, the fragmented consciousness (59).

La postura de Anna frente a su propia teoría se devela desde la primera parte de la historia, lo que sugiere una autoconsciencia del proceso creativo y define la naturaleza del ejercicio: "Yet I am incapable of writing the only kind of novel which interests me: a book powered with an intellectual or moral passion strong 
enough to create order, to create a new way of looking at life" (59). La paradoja, una vez más, es que nos cuenta de su incapacidad y decisión de no escribir una novela, escribiendo una.

El rasgo autobiográfico más sobresaliente en la narración de Wulf se ejemplifica en la definición indentitaria dividida en varias secciones, como madre, amiga, pareja, escritora, activista, crítica y, claro, como mujer. Sin que ninguna categoría pese más que otra, Anna intenta reconciliarlas a través de su escritura, que nos recuerda al acto de "ser" después de haberse escrito (a sí misma). "Scribo, ergo sum" es la oración con la que Kellman (1980: 5) resume el acto autobiográfico de la self-begetting novel, pues no se trata sólo de escribir la vida pasada, sino, por una parte, de revivirla en la escritura y, por otra, de tomar una postura moral respecto a quien se era en ese momento y quien escribe desde el presente: “when I read my notebook I didn't recognise myself. Something strange happens when one writes about oneself That is, one's self direct, not one's self projected. The result is cold, pitiless, judging" (545).

La tensión de Anna con la acumulación caótica y, para ella, sin sentido de su experiencia la lleva a un análisis autocrítico y desgastante que termina en un bloqueo creativo y en un fracaso temporal: "I am a person who continually destroys the possibilities of a future because of the numbers of alternative viewpoints I can focus on the present" (620). Esta lucha está anunciada desde las primeras páginas: "Anna grimaced, acknowledging failure. She could not learn languages, and was too self-conscious ever to become somebody else" (5). La duda de ser quien se cree que es y la incapacidad de asir un significado propio se narran antes de que Anna introduzca el metatexto en los cuadernos y sugieren una preocupación temática autobiográfica que germinará en la identidad artística. Así, la naturaleza teórica de Anna y de su novela se retrata en forma y tema durante todo el libro, pero se anuncia desde el principio: “'There you are, said Molly [amiga de Anna a ésta], antagonistic; 'but I'm not a theoretical type. You always do that-faced with something you start making up theories'” (11).

En una suerte de convergencia entre el género dramático y el narrativo, nos encontramos en The Golden Notebook con indicaciones en la disposición de una escena o con la descripción de la parte que a continuación vamos a leer. $\mathrm{Al}$ igual que los intertítulos y los corchetes, que establecen un cambio en la voz 
narrativa y en el contrato de lectura, las acotaciones denotan una voz consciente de su público y un interés en que la(s) novela(s) se lean a través de una lente específica; es decir, funcionan como instrucciones: constriñen la imaginación de la lectora respecto de lo que está leyendo y crean un efecto dramático, el de conocer en su esplendor la escena que se está retratando. Así lo vemos, por ejemplo, en el principio de "Free Women: 1", donde debajo del intertítulo principal se lee "Anna Meets Her Friend Molly in the Summer of 1957 after a Separation" (3), seguido de la presentación de la escena: "The two women were alone in the London flat" (3). En un principio, la necesidad de estas acotaciones resulta en realidad oscura, pues pensaríamos que las herramientas narrativas descriptivas de una novela funcionarían con mayor naturalidad. Sin embargo, el uso de este tipo de paratextos indicativos queda justificado cuando se reconoce el ensayo de diversos géneros literarios en la configuración de una novela.

"La novela es un género complejo - afirma Brioschi-que acoge dentro de sí las formas de otros géneros, haciéndolos reaccionar entre sî" (1988: 238). Como parte de un ejercicio autoral creativo, la introducción de acotaciones casi teatrales es, probablemente después del género autobiográfico que guía el relato, el ejemplo más directo de una práctica narrativa consciente de su artificio. Está sugerida desde el índice - cada parte contiene su acotación abajo del intertítulo- y organiza la novela en partes perfectamente definidas: "Two visits, some telephone calls and a tragedy"; "Tommy adjusts himself to being blind while the older people try to help him"; "Anna and Molly influence Tommy for the better. Marion leaves Richard. Anna does not feel herself" (V). El guiño al arte dramático escogido por Anna la novelista, no obstante, no es sólo un homenaje a Molly, amiga actriz y otro de los tantos yos de Anna, sino al arte de interpretar a alguien más. La práctica artística de Anna, después de todo, es también un ejercicio identitario de probar ser distintas voces a través de la narración, es decir, a través de las máscaras literarias: "I was a character in my own play" (241), apunta Anna al contar un sueño.

$\mathrm{Al}$ ser un ensayo, un ejercicio experimental, la práctica creativa de Anna no está exenta de errores. Uno de los fracasos que notamos en la intervención de los paratextos indicativos es que esta voz, que modula todo dentro de The Golden Notebook como novela y que da la impresión de hacer aclaraciones objetivas y 
necesarias de los cuadernos, también hace juicios de valor y toma posturas que no le corresponderían si fuera, en efecto, una narradora heterodiegética, y que sugieren un grado inevitable de subjetividad: "[The yellow notebook looked like the manuscript of a novel, for it was called The Shadow of the Third. It certainly began like a novel]" (161, cursivas mías).

En suma, los elementos paratextuales que toman la forma de intertítulos, indicaciones entre corchetes y acotaciones casi teatrales, y el metacomentario de Anna en los mismos cuadernos constituyen los instrumentos de su experimento creativo: explorar las posibilidades del artificio cuando se intenta plasmar la experiencia vivida. Paradójicamente, retratan la mayor característica de dicho proceso - la relación tensa entre la escritora y el lenguaje-, mientras que le conceden una unidad arquitectónica a la novela. Los paratextos, además, tienen el "efecto inevitable [y consciente] de constituir a [la heroína-narradora] en instancia no sólo narrativa, sino también literaria [...] Ya no se trata solamente, como en el caso de Lazarillo, de un personaje que cuenta su vida por escrito, sino de un personaje que se hace escritor y hace de su relato un texto literario" (Genette, 2001: 257). Para Anna, la literatura es el espacio de las posibilidades humanas donde la experiencia adquiere valor y significado y nos sugiere que el lenguaje literario es la forma más acertada — si bien no definitiva- de recontar la experiencia.

\section{Referencias bibliográficas}

Brioschi, Franco; Di Girolamo, Costanzo. (1988). “Formas literarias”. En Introducción al estudio de la literatura (pp. 97-108). Ariel.

Fowles, John. (2010). The French Lieutenant's Woman. Little, Brown and Company.

Genette, Gérard. (2001). Umbrales (Susana Lage, Trad.). Siglo XXI.

Hutcheon, Linda. (1980). Narcissistic Narrative. The Metafictional Paradox. Wilfrid Laurier University Press. 
Kellman, Steven G. (1980). The Self-begetting Novel. Columbia University Press.

Lessing, Doris. (1999a). "Introduction 1993", "Introduction 1971". En The Golden Notebook (pp. vII-XXVII). Harper Perennial.

Lessing, Doris. (1999b). The Golden Notebook. Harper Perennial.

Woolf, Virginia. (2002). “The Modern Essay”. En Project Gutenberg Australia. 